Rey. Elev. Méd. vét. Pays trop., 1974, 27 (1) : 53-56

\title{
Note sur les helminthes (et quelques autres parasites) du dromadaire en Ethiopie
}

\author{
par P. DAYNES (*) et D. RICHARD (*) \\ (avec la collaboration technique de M. GUEBRE NEGUS) $\left(^{* *}\right)$
}

\begin{abstract}
RESUME
Les auteurs rapportent les résultats de travaux effectués sur le Dromadaire en Ethiopie. Ils signalent 10 espèces d'helminthes, la cysticercose, l'hydatidose, un cstridé et rappellent la présence de 10 espèces de tiques, d'un trypanosome, d'un acarien et d'une coccidie.
\end{abstract}

\section{INTRODUCTION}

Il y aurait environ 800.000 dromadaires (Camelus dromedarius) en Ethiopie selon les statistiques 1965-1971 (1) mais leur nombre réel doit se situer en fait autour du million.

Ces animaux se rencontrent essentiellement en Erythrée et dans l'Est du Wollo et du Tigré, dans le Harrar, dans le Sud du Balé et du Sidamo, c'est-à-dire dans des zones de climat tropical subdésertique.

Le dromadaire est utilisé pour son travail comme animal de bât, pour son lait et pour sa viande, bases d'alimentation protidique de toute une population. Il est en outre l'objet d'un important commerce d'exportation vers la Somalie et l'Arabie.

Il nous a donc semblé utile d'entreprendre une étude de la parasitologie de cet animal et nous exposons ici les premiers résultats obtenus en 1973.

\section{GENERALITES}

Du point de vue parasitologie des animaux domestiques en Ethiopie, il existe un bon nom-

(*) French Veterinary Mission, P.O. Box 1053, Addid Abeba, Ethiopie.

(w*) Imperial Veterinary Institute, P.O. Box 19, Debre-Zeit, Ethiopie. bre de travaux épars et, du point de vue synthétique, deux rapports importants : celui de BERGEON (2) après un séjour de 2 ans, et celui de GRABER (7) après une mission d'expertise de 3 mois. Ces deux rapports font la synthèse des connaissances acquises en Ethiopie du point de vue parasitologie vétérinaire.

En ce qui concerne le dromadaire, BERGEON ne relève qu'un parasite : "Trypanosoma evansi was found in dromedaries in Ogaden ».

GRABER en cite davantage tout en relevant le peu de travaux effectués: Sur 335 références de son rapport, 2 seulement se rapportent au dromadaire en Ethiopie : PRICOLO (9) signale Dipetalonema evansi; CHIODI (4) signale Anthostrongylus somaliensis (= Impalaia nudicollis) et des kystes hydatiques d'echinocoque (Echinococcus polymorphus) dans le foie et dans le poumon.

Pour sa part GRABER (7) étudiant 11 animaux reconnaît des strongles digestifs dans 11 cas et des trichures dans 4 cas. II diagnostique les espèces suivantes: Haemonchus longistipes, Oesophagostomum columbianum, Impalaia nudicollis, Trichuris globulosa, ainsi qu'Eimeria noelleri.

Compte tenu de l'importance du dromadaire en Ethiopie, nous nous sommes attaché, dans 
un premier temps, à en rechercher les principaux parasites capables d'exercer une influence défavorable sur son élevage.

\section{MATERIEL ET METHODE}

\section{- Coproscopies}

Nous avons effectué 351 coproscopies dont 262 dans la Région du Neguelé Borana au Sud et au Sud-Est de cette ville (196 en août, septembre et 82 en novembre) et 73 dans le Harrar, le Tigré et l'Erythrée (décembre).

La méthode utilisée a été la méthode multivalente d'enrichissement par sédimentation appliquée parfois à des prélèvements frais, la plupart du temps à des prélèvements formolés.

Les formes parasitaires reconnues ont été classées en strongles digestifs, sans diagnose plus précise, en Strongyloïdes, Trichures, ciliés et embryophores de Cestodes.

\section{- Autopsies}

Nous avons effectué 10 autopsies complètes et 10 études partielles de tubes digestifs, parfois d'autres organes.

3 dromadaires ont été étudiés à Dire Dawa et 17 à Neguele-Borana.

Les études partielles ont intéressé soit une partie du tube digestif, soit un seul organe (cas du dromadaire $\mathrm{n}^{\circ} 12$ par exemple dont seul le cœur a été vu), soit des Cestodes ramassés sur le sol après vidange du tube digestif lors de l'abattage.

\section{RESULTATS}

\section{- Coproscopies}

Les résultats de coproscopies apparaissent dans le tableau $\mathrm{n}^{0} \mathrm{I}$.

N'apparaissent pas les embryophores de Cestodes, rencontrés rarement.

\section{- Autopsies}

Nous donnons les résultats obtenus sous forme de tableau ce qui, concernant les autopsies complètes, permet de se faire une idée précise du polyparasitisme rencontré (tableau $\mathrm{n}^{\circ}$ II).

\section{DISCUSSIONS}

Les coproscopies effectuces montrent la grande fréquence des Strongles digestifs (sensu lato) et la relative fréquence des Trichures. Les ciliés se rencontrent dans une coproscopie sur deux alors qu'on n'a rencontré aucun œuf de Trématode.

Dans tous les cas d'autopsie complète, on a pu mettre en évidence au moins deux helminthes différents, un Cestode et un Nématode, dans 3 cas sur 10 et quatre espèces d'helminthes ou plus dans 7 cas sur 10 . Aucun Trématode n'a été trouvé lors de ces autopsies.

$\mathrm{Si}$ ces dernières confirment les coproscopies en ce qui concerne la fréquence des strongles digestifs, on voit par contre que les Cestodes sont plus facilement rencontrés lors d'autopsies. On peut noter également que nous n'avons pas rencontré de Strongyloïdes lors des raclages de muqueuse alors que les coproscopies en montrent chez un animal sur dix environ. Le petit nombre d'autopsies effectuées ne permet cependant pas de faire de pourcentage d'infestation.

\section{AUTRES PARASITES RENCONTRES}

En ce qui concerne les parasites autres que les helminthes, il faut signaler la très grande fréquence des larves de Cephalopsis titillator, cstridé parasite du nasopharynx et des sinus frontaux, rencontrées en grande quantité lors de toutes nos autopsies ou études partielles, soit dans 23 cas sur 23 , dont 20 dans le Borana.

Les tiques rencontrées sont d'après BERGEON et BALIS (3) (1974) Rhipicephalus pulchellus, $R$. Simus, $R$. parvus, Amblyomma variegatum, $A$. lepidum, $A$. gemma, Hyalomma excavatum, $H$. dromedarii, $H$. impeltatum et $H$. rufipes.

Le seul trypanosome, diagnostiqué déjà par BERGEON (2), est Trypanosoma evansi.

Du point de vue des gales, nous avons observé un Sarcoptes scabiei var. cameli sur un dromadaire à la clinique vétérinaire de DireDawa.

Notons enfin la fréquence des infusoires dans plus de 50 p. 100 des coproscopies effectuées 
Retour au menu

TABLEAU $\mathrm{N}^{\circ} \mathrm{I}$

Résultats des coproscopies

\begin{tabular}{|c|c|c|c|c|c|}
\hline & & S.D. & Str. & T. & Ci1, \\
\hline \multirow{2}{*}{$\begin{array}{c}I \\
(196)\end{array}$} & $\mathrm{N}$ & 163 & 23 & 47 & 81 \\
\hline & p. 100 & 83 & 12 & 24 & 41 \\
\hline \multirow{2}{*}{$\begin{array}{l}\text { II } \\
(82)\end{array}$} & $\mathrm{N}$ & 74 & 7 & 9 & 75 \\
\hline & p. 100 & 90 & 8,5 & 11 & 91 \\
\hline \multirow{2}{*}{$\begin{array}{l}I+I I \\
(278)\end{array}$} & $\mathrm{N}$ & 237 & 30 & 56 & 156 \\
\hline & p. 100 & 85 & 11 & 20 & 56 \\
\hline \multirow{2}{*}{$\begin{array}{l}\text { III } \\
\text { (73) }\end{array}$} & $\mathrm{N}$ & 30 & 2 & 5 & 21 \\
\hline & p.100 & 41 & 3 & 7 & 29 \\
\hline \multirow{2}{*}{$\begin{array}{c}I+I I+I I I \\
\quad(351)\end{array}$} & $\mathrm{N}$ & 267 & 32 & 61 & 177 \\
\hline & P. 100 & 76 & 9 & 17 & 50 \\
\hline
\end{tabular}

I = 1re série - 196 coproscopies - Neguele-Borana - août-septembre.

II $=2^{e}$ série - 82 coproscopies - Neguele-Borana - novembre.

III = $=3 \mathrm{e}$ série - 73 coproscopies - Harrar/Tigre/Erythrée - décembre.

S.D. = Eufs de Strongles digestifs.

Str. = Eufs de Strongyloïdes.

T. $\quad=$ Eufs de Trichures.

Cil. = Présence de Ciliés.

N. $=$ Nombre d'animaux reconnus parasités.

p. $100=$ Pourcentage d'animaux reconnus parasités.

TABLEAU N*II

Helminthes récoltếs chez le dromadaire en Ethiopie en 1973

\begin{tabular}{|c|c|c|c|c|c|c|c|c|c|c|c|c|c|c|c|c|c|c|c|c|}
\hline & \multicolumn{10}{|c|}{ Autopgies complètes } & \multicolumn{10}{|c|}{ Autopsies partielles } \\
\hline Lieu & \multirow{2}{*}{\multicolumn{2}{|c|}{$\begin{array}{l}\text { Diré } \\
\text { Dawa } \\
\text { Nov, }\end{array}$}} & \multicolumn{8}{|c|}{ Neguele-Bor ana } & $\begin{array}{l}\text { Dire } \\
\text { Dewa }\end{array}$ & \multicolumn{9}{|c|}{ Neguele-Borana } \\
\hline Date & & & \multicolumn{8}{|c|}{ Oet. } & Nov & Hil & \multicolumn{4}{|c|}{ Août } & \multicolumn{4}{|c|}{ Oct. } \\
\hline $\mathbb{N}^{*} \mathrm{~d}^{\prime}$ ordre & I & 2 & 3 & 4 & 5 & 6 & 7 & 8 & 9 & 10 & 11 & 12 & 13 & 14 & 15 & 16 & 17 & 18 & 19 & 20 \\
\hline Moniezia exparisa & + & & & & & & & & & & & & & & & & + & + & + & \\
\hline Moniexia sp. & & & + & & & & + & + & + & + & & & & & & + & & + & & + \\
\hline Stilesia vittata & + & + & & & + & & & + & & & & & & & & & & & & \\
\hline Avitelline centmipunctata & + & & + & + & & & & & & & & & & & & & + & & & \\
\hline Hydatidose & & & & & P.P. & & & & & & R. & & & & P. & & & & & \\
\hline Cysticercose & & & & & & & & & & & & c. & & & & & & & & \\
\hline Triohumis globutosa & + & & & & + & + & & & + & & & & + & + & & & & & & \\
\hline Triahuria ovis & & & & & & & + & & & & & & & & & & & & & \\
\hline Trichuria sp. & & + & & + & & & & + & & & & & & & & & & & & \\
\hline Hasmonohus oontortus & & & & + & & + & + & + & + & & & & & & & & & & & \\
\hline Trichostrongt tus probolurus & + & & + & & & + & + & & & + & & & & & & & & & & \\
\hline Impalaia somalienais & + & & + & + & & + & + & + & + & & & & & & & & & & & \\
\hline Bunostomm trigonocephalum & & & & & & + & & & & & & & & & & & & & & \\
\hline Parabronema okrjabini & & & + & & & & & & & & & & & & & & & & & \\
\hline
\end{tabular}

F a foie; $P$ - poumon; $R$ = Rate; $C$ = coeur.

Rectification : au lieu de Impalaia somaliensis, lire Anthostrongylus somalilensis. 
et l'existence de Eimeria noelleri, rapportée par GRABER (7).

L'infestation par hirudinacées n'a été signalée aucune fois dans les régions visitées.

\section{CONCLUSIONS}

Cette première étude du parasitisme du dromadaire nous a permis de mettre en évidence un certain nombre d'helminthes non encore signalés en Ethiopie chez cette espèce animale.
Elle a montré une fois de plus la valeur et les limites de la seule coprologie dans un but d'enquête épidémiologique.

Elle montre à l'évidence le rôle pathologique potentiel du parasitisme dans l'élevage d'une espèce animale dont le rôle économique est loin d'être négligeable.

Les résultats obtenus invitent à poursuivre le travail entrepris en ne se limitant pas d'ailleurs aux seuls helminthes.

\title{
SUMMARY
}

\begin{abstract}
Note about helminths (and some other parasites) of dromedary in Ethiopia
\end{abstract}
The results of works done on one-humped camel in Ethiopia are reported. The authors point out the presence of ten species of helminths, cysticercosis, hydatidosis, and one oestridae and they recall the presence of ten tick species, one trypanosoma, one mite and one coccidia.

\section{RESUMEN} Nota sobre los helmíntos (y algunos otros parásitos) del dromedario
en Etiopia

Los autores notan los resultados de investigaciones efectuados sobre el dromedario en Etiopia. Señalan diez especies de helmíntos, la cisticercosis, la hidatidosis, un éstrido y recordan la preséncia de diez especies de garrapatas, de un tripanosomo, de un ácaro y de una coccidia.

\section{BIBLIOGRAPHIE}

1. A review of animal Health and livestock productivity factors in Ethiopia, 1965-1971. - Addis-Abeba, The department of Veterinary Services. I.E.G. Ministry of Agriculture, 1972.

2. BERGEON (P.). Report to the Government of Ethiopia on Veterinary Parasitology Survey. Rome, F.A.O., 1968. (Publ. F.A.O. no TA - 2458), 38 p.

3. BERGEON (P.) et BALIS (J.). Contribution à l'étude de la répartition des tiques dans l'Empire d'Ethiopie (enquête effectuée de 1965 à 1969). Rev. Elev. Méd. vét. Pays trop., 1974 (sous presse).

4. CHIODI (V.). Brevi cenni nosografici dell Ethiopia. G. Ital. Mal. esot. Trop. Ig. Colon, 1936, 9 (8) : 157-163.

5. GRABER (M.) et GRUVEL (J.). Etude des agents des myiases des animaux domestiques et sauvages
d'Afrique équatoriale. Rev. Elev. Méd. vét. Pays trop., 1964, 7 (3) : 535-554.

6. GRABER (M.), TABO (R.) et SERVICE (J.). Enquête sur les Helminthes du dromadaire tchadien. Etude des Strongyloses gastro-intestinales et de l'Hémonchose à Haemonchus longistipes. Rev. Elev. Méd. vét. Pays trop., 1967, 20 (2): 227-254.

7. GRABER (M.). Helminthes et helminthoses des animaux domestiques et sauvages d'Ethiopie. Rapport de Mission. Maisons-Alfort, I.E.M.V.T., 1973, $206 \mathrm{p}$.

8. MALEK (E.). Check-list of Helminth - Parasites in Sudan. Indian vet. J., 1959, 36 (6) : 284-285.

9. PRICOLO (A.). Larves de filaires dans le sang de chameaux tunisiens et de l'Erythrée. Zentbl. Bakt. Parasit. Kde, Abt. I., 1913, 71: 478-479. 